\title{
Size Effect in the In-plane Electrical Resistivity of Very Thin Graphite Crystals
}

\author{
Y.Ohashi, T.Koizumi*, T.Yoshikawa**, T.Hironaka and K.Shiiki
}

(Received June 30, 1997, Accepted November 24, 1997)

\begin{abstract}
In order to investigate the effect of film thickness of thin graphite crystals on the electrical properties, we prepared thin graphite films by cleaving a kish graphite $(\mathrm{KG})$ crystal with the rrr value of 32.3 . The values of the thickness of the graphite films were ranging from around 300 to $1000 \AA$. Keeping these graphite films free from strain, we measured the temperature dependence of the in-plane resistivity between liquid helium and room temperatures. The experimental results could be expressed by a simple two band model and the Sugihara's theory for lattice vibration in thin-carbon films. By using these expressions, we estimated the overlap energy $E_{0}$ of conduction and valence bands for the thin graphite films with various thicknesses. It was found that $E_{0}$ decreases with decreasing film thickness. We also estimated the reciprocal of the relaxation time due to lattice defects or surface scattering $1 / \tau_{\mathrm{i}}$ and that due to lattice vibration $1 / \tau_{\mathrm{i}}$ for the thin films with various thicknesses. Consequently, it was found that the effect of $1 / \tau_{\mathrm{i}}$ increases rapidly, as the film thickness decreases.
\end{abstract}

KEYWORDS : graphite, thin-film, resistivity, size effect, band overlap energy

\section{INTRODUCTION}

According to the Slonczewski - Weiss - McClure (SWMcC) energy band model, the three dimensional graphite should be semimetal because the valence band overlaps slightly to the conduction band ${ }^{1 / 2)}$, while the two dimensional graphite should be zero band gap semi-conductor because the valence band and the conduction band touch together just at the Fermi leve ${ }^{13)}$. Moreover, the graphite dilayer density of state at the Fermi level has been predicted to be much smaller than that for the bulk crystal $^{4)}$. Therefore, the electron energy band structure of very thin graphite crystals is expected to be different from that of bulk crystals, that is, the degree of the overlap of these two bands will depend on the film thickness. Moreover, the bands overlap should be reflected in the carrier concentrations. In fact, some kinds of carbon fibers have been treated as quasi-two dimensional carbon materials. The electrical properties of these materials were measured under the various conditions ${ }^{5)-8)}$. Especially, the temperature dependence of the electric resistivity was discussed in relation to the effect of heat treatment temperature (HTT) 5) ,7) ,8). HTT, however, has an influence on both basal plane stackings and the crystalline growth along the basal plane, simultaneously. Therefore, it will be necessary to measure the electrical properties of thin graphite films with good crystal perfection, only by changing the thickness.

The measurements of the electrical resistivity have already been carried out for kish graphite (KG) films with various thicknesses, and it has been reported that the carrier concentrations of very thin crystals are different from those of bulk crystals $\left.{ }^{9)}, 10\right)$. However, two subjects were found to be improved. In the first place it was pointed out that the thin films cemented on the substrates with the epoxy resin might be distorted by changing the temperature and this distortion should have an effect on their electric resistivity. In the second place the residual resistivity ratio (rrr) values of the bulk KG crystals had not been measured before cleaving. The value of $\mathrm{rrr}$ is the ratio of the resistivity measured at room temperature to that at $4.2 \mathrm{~K}$. The quality of the graphite materials is evaluated by the rrr value.

In this study, we prepared the graphite specimens of thickness ranging from $290 \AA$ to $1110 \AA$ by cleaving a KG crystal with high crystallinity improving on two points mentioned above. The temperature dependence of the electric resistivity was measured in the temperature range between $4.2 \mathrm{~K}$ and room temperature. A simple two band (STB) model ${ }^{11)}$ and the effect of the lattice vibration ${ }^{12)}$ were applied to the experimental results, and then the values of the band overlap energy were estimated for the thin graphite crystals with various film thicknesses.

\section{EXPERIMENTAL}

All the specimens measured in this study were made of an original bulk crystal of KG. The rrr value of the crystal was 32.3. The graphite crystal used in this study can be judged to be a good one.

We prepared specimens by the following procedure : we fixed the graphite crystal on a glass plate covered with a doublesided adhesive tape, applied a small hard rubber block covered with another double-faced adhesive tape on the crystal surface to cleave, and repeated this operation untill the crystal turned translucent. After washing the crystal in organic solvent to remove from double-faced adhesive tape, we just put the graphite films on quartz plates and did not fix them, in order to keep the specimens free from strain caused by varying temperature.

The sample thickness was determined from the measurement

Faculty of Science and Technology, Keio University : 3-14-1 Hiyoshi, Kouhoku-ku, Yokohama 223, Japan

* Toyo Corporation : 3-26-9 Yushima, Bunkyo-ku, Tokyo 113, Japan

** Matsushita Communication Industrial CO., LTD. : 4-3-1,Tsunashima-Higashi, Kohoku-ku, Yokohama 223, Japan 


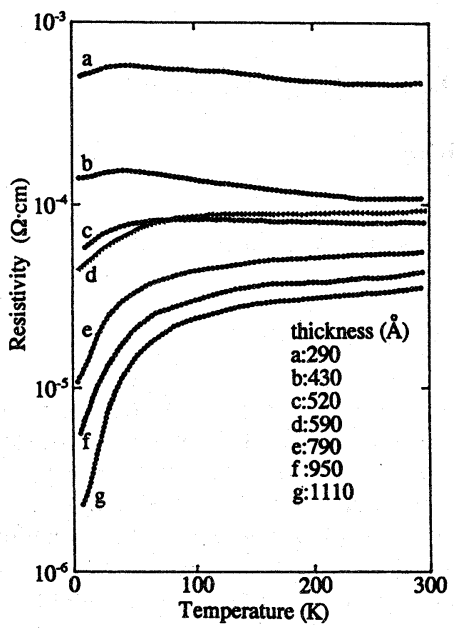

Fig.1 Temperature dependence of in-plane resistivity for thin films with various thickness prepared by cleaving the kish graphite with the rrr value of 32.3

of transmitted light intensity at the wavelength of He-Ne laser by referring to a theoretical relationship between transmission and thickness, which had been calculated by taking $\eta$ (the refractive index) $=2.2$ and $\kappa$ (the extinction coefficient) $=$ 1.4 at normal incidence for graphite ${ }^{13)} \mathrm{A}$ uniform part in the film was worked into a bridge type specimen under a binocular microscope ${ }^{9)}$, where the deviation of thickness from the average value was about $20 \AA$ at most and the surface area was larger than $0.05 \mathrm{~mm}^{2}$. Silver fine wires $0.1 \mathrm{~mm}$ in diameter were fixed to the specimen with conductive silver coating material as electrodes. The resistivity was measured with conventional four terminal method. The temperature was measured from $4.2 \mathrm{~K}$ to room temperature using an $\mathrm{Au}-\mathrm{Fe}$ versus Chromel thermocouple.

\section{RESULTS}

Fig.1 shows the in-plane electric resistivity $\rho$ of thin graphite crystals with various film thicknesses as a function of temperature $T$. Roughly speaking, the thinner the crystal, the larger the resistivity in the whole range of temperature measured. The lattice defects may be produced by the cleaving operation, but the film thickness did not depend on the number of cleaving operation to make thin films. Therefore, it can not be expected that the thinner crystals have more defects. The temperature dependence of the resistivity of crystals thinner than $500 \AA$ reveals a peak at around several tens degree Kelvin. This tendency of $\rho-T$ curves is qualitatively consistent with the result obtained before ${ }^{9)}$

\section{ANALYSIS AND DISCUSSION}

The temperature dependence of resistivity $\rho$ defined by Eq. (1) depends on total carrier density $\mathrm{n}$ and mean relaxation time $\tau$

$$
\rho=\frac{1}{\sigma}=\frac{\mathrm{m}^{*}}{\mathrm{ne}^{2}} \cdot \frac{1}{\tau}
$$

where $\mathrm{m}^{*}$ and e represent the mean effective mass and the electric charge of carriers, respectively.

In thin-carbon film, the reciprocal of the mean relaxation time due to the scattering by the Rayleigh-wave phonons is proportional to temperature reported theoretically by Sugihara ${ }^{12)}$. Assuming that the theory can be applicable to our case, Eq. (2) is obtained, where $1 / \tau_{\mathrm{i}}$ and $1 / \tau_{\mathrm{L}}$ are the relaxation rates due to lattice defects and the lattice vibration respectively, and the latter is proportional to $T$

$$
\frac{1}{\tau}=\frac{1}{\tau_{\mathrm{i}}}+\frac{1}{\tau_{\mathrm{L}}}=\frac{1}{\tau_{\mathrm{i}}}+\mathrm{A} T
$$

where $A$ is constant. On the other hand, using the STB model ${ }^{11)}$, $n$ is approximately given by Eq. (3)

$$
\frac{n}{2}=n_{\mathrm{e}}=n_{\mathrm{h}}=\frac{8 \pi m^{*}}{h^{2} c_{\mathrm{o}}} k T \ln \left(1+\exp \frac{E_{0}}{2 k T}\right)
$$

where $n_{e}$ and $n_{h}$ are densities of electrons and holes respectively, h the Plank's constant, $k$ the Boltzman's constant, $\mathrm{c}_{\mathrm{o}}$ the lattice constant. Using Eqs. (2) and (3) , Eq. (1) is written

$$
\rho=\frac{h^{2} c_{\mathrm{o}}}{16 \pi e^{2}} \frac{1}{k T \cdot \ln \left(1+\exp \frac{E_{0}}{2 k T}\right)}\left(\frac{1}{\tau_{i}}+A T\right)
$$

Especially, it should be noted that the resistivity $\rho_{0}$ at zero $\mathrm{K}$ depends on both $\tau_{\mathrm{i}}$ and $E_{0}$ as follows :

$$
\rho_{0} \equiv \frac{h^{2} c_{\mathrm{o}}}{8 \pi e^{2} E_{0} \tau_{\mathrm{i}}}
$$

In order to expand the temperature dependence of the resistivity of thin films, $\rho$ was normalized with the value at the liquid helium temperature as shown in Figs.2 (a) and (b) , which can correspond to Eq. (6) derived from Eqs. (4) and (5)

$$
\frac{\rho}{\rho_{0}}=\frac{E_{0}}{2 k T \ln \left(1+\exp \frac{E_{0}}{2 k T}\right)}\left(1+\tau_{i} A T\right)
$$

The solid curves in Figs.2 (a) and (b), which were calculated by using Eq. (6), represent the best fits to the experimental data. In addition, by using the value $E_{0}$ and the experimental data $\rho_{\mathrm{T}} / \rho_{4,2 \mathrm{~K}}$, we can ascertain that the temperature dependence of $1+\tau_{i} A T$ for each specimens will be valid as shown in Fig.3. This tendency is qualitatively consistent with the Sugihara's theory, though the phonon scattering rate isn't proportional to $d^{-2}$, where $d$ is the sample thickness ${ }^{12)}$. The values of $E_{0}$ and $\tau_{\mathrm{i}} A$ for various film thicknesses are shown in Fig.4 and Fig.5, respectively. As shown in Fig.4, the energy $E_{0}$ decreases with decreasing film thickness gradually and seems to have a small value at the thickness thinner than about $500 \AA$. The ratio $\tau_{\mathrm{i}} A$ of two relaxation times $1 / \tau_{\mathrm{i}}$ and $1 / \tau_{\mathrm{L}}$ at constant temperature decreases with decreasing film thickness, and at the thickness thinner than about $500 \AA$, $\tau_{\dot{j}} A$ also has a small value as shown in Fig.5. On the other hand, each value of $1 / \tau_{\mathrm{L}}$ and $1 / \tau_{\mathrm{i}}$ increases with decreasing film thickness as shown in Fig.6. Especially, $1 / \tau_{\mathrm{i}}$ of thin films thinner than about $500 \AA$ seems to 

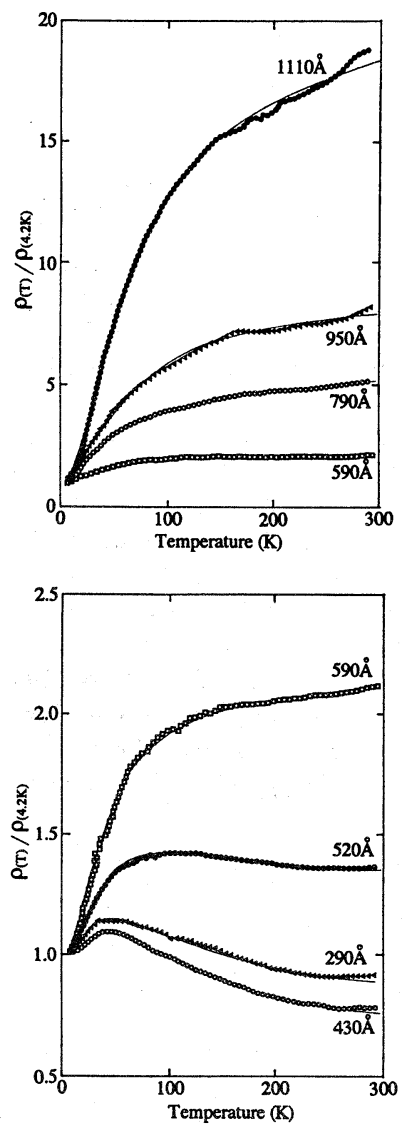

Fig.2 Resistivity normalized with the value at $4.2 \mathrm{~K}$ as a function of temperature for various film thickness of the $\mathrm{KG}$ specimens, (a) thickness from $590 \AA$ to $1110 \AA$, (b) thickness from $290 \AA$ to $590 \AA$. The curve of $590 \AA$ in (a) is the same as that in (b). The solid curves, which were calculated by using the model described in the text, represent the best fits to the experimental data. $290 \AA$ to $590 \AA$

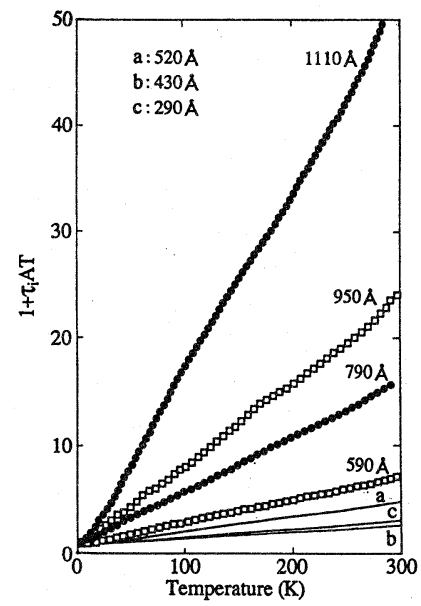

Fig.3 Ratio $1+\tau_{\mathrm{i}} A T$ of two relaxation times $\tau_{\mathrm{i}}$ and $\tau_{\mathrm{L}}$ versus temperature for various thickness.

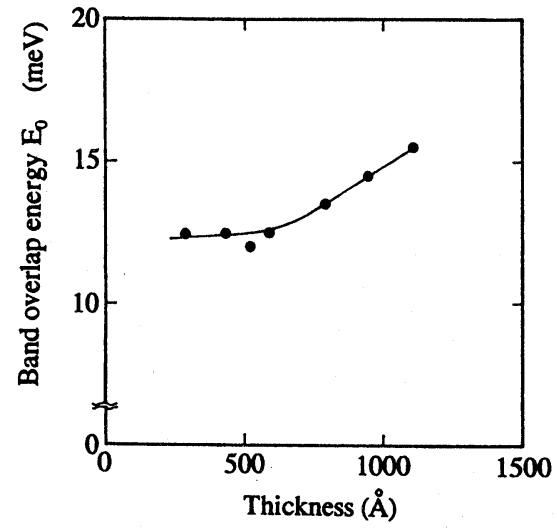

Fig.4 Band overlap energy $E_{0}$ as a function of film thickness.

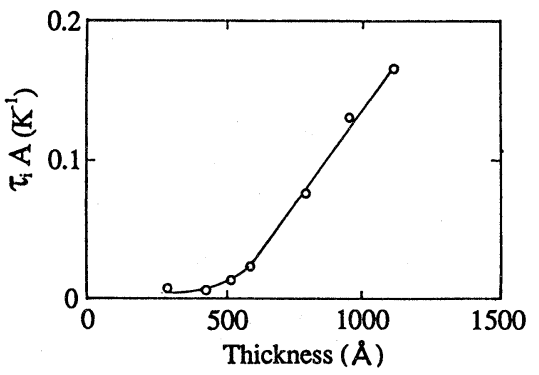

Fig.5 Ratio $\tau_{\mathrm{i}} A$ of two relaxation times $\tau_{\mathrm{i}}$ and $\tau_{\mathrm{L}}$ at constant temperature as a function of thickness.

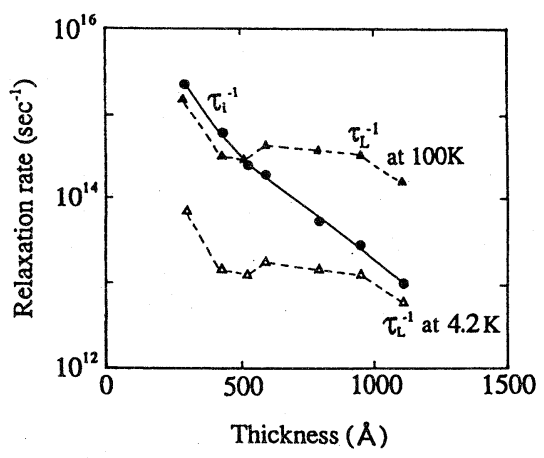

Fig.6 Relaxation rate $1 / \tau_{\mathrm{L}}$ and $1 / \tau_{\mathrm{i}}$ as a function of thickness.

be predominant even in the high temperature region. In order to investigate the carrier concentrations and the relaxation times of thin graphite crystals separately, measurements of the galvanomagnetic properties will be required.

\section{CONCLUSION}

Thin graphite crystals were prepared by cleaving a KG crystal with the rrr value of 32.3 and were kept free from strain. The thickness of thin graphite crystals was ranging from around 300 to $1000 \AA$. The temperature dependence of the in-plane resistivity 
of these graphite films was measured at temperatures between $4.2 \mathrm{~K}$ and room temperature. Applying the STB model and the Sugihara's theory for lattice vibration in thin-film carbon on the experimental results, we obtained the overlap energy $E_{0}$ of conduction and valence bands for the graphite films with various film thicknesses. It was found that $E_{0}$ decreases with decreasing thickness and approaches to a small value for the films thinner than $500 \AA$. Furthermore, it was found that the effect of $1 / \tau_{i}$ increase raidly, as the film thickness decreases.

\section{ACKNOWLEDGMENTS}

The authors wish to thank Professor Hishiyama of Musashi Institute of Technology for kindly supplying a valuable KG crystal with the rrr value of 32.3. A part of this work was supported by the grant of Sumitomo Kinzoku Kogyo Co Ltd.

\section{REFERENCES}

1) J.C.Slonczewski and P.R.Weiss, Phys. Rev. 109, (1958) 272-279.

2) J.W.McClure, Phys. Rev. 108, (1957) 612-618.

3) P.R.Wallace, Phys. Rev. 71, (1947) 622-634.

4) S.B.Trickey, F.Muller-Plathe, G.H.F.Diercksen and J.C.Boettger,Phys.Rev.B 45, (1992- II) 4460-4468

5) I.L.Spain, K.J.Volin, H.A.Goldberg and I.L.Kalnin, Solid State Comm. 45, (1983) 817-819.

6) L.D.Woolf, J.Chin, Y.R.Lin-Liu and H.Ikezi, Phys. Rev. $B$ 30, (1984) 861-869

7) M.Z.Tahar, M.S.Dresselhaus and M.Endo, Carbon 24, (1986) 67-72.

8) A.N.Chuvyrov, Yu.A.Lebedev, V.M.Kornilov and Salimgareeva, Sov. Tech. Phys. Lett. 16, (1990) 141-142.

9) Y.Fujibayashi,J. Phys. Soc. Japan 34, (1973) 989-993.

10) Y.Fujibayashi and S.Mizushima, J. Phys.Soc.Japan 34, (1973) 281.

11) C.A.Klein, J. Appl. Phys. 35, (1964) 2947-2957.

12) K.Sugihara, Phys. Rev. B 37, (1988) 7063-7069.

13) S.Ergun and J.T.McCartney, Proc. 5th Conference on Carbon 2 (1963) pp 167-173, Pergamon Press. 